\title{
Orbital Cellulitis with Exudative Retinal Detachment
}

\author{
Pooja Karki ${ }^{1}$, Manish Pandey ${ }^{1}$
}

${ }^{1}$ Nepal Eye Hospital, Kathmandu Nepal.

\section{ABSTRACT}

Exudative retinal detachment occurs extremely rarely with orbital cellulitis. TB can affect majority of the structures of the eye with marked variability of the lesions. We herein report a 40 year old male who presented with acute left eye protrusion with sudden diminution of vision. On examination there was proptosis of the left eye with restricted extraocular movements. Exudative retinal detachment with subretinal abscess was present in the same eye. Magnetic Resonance Imaging (MRI) of brain showed right sided frontal lobe lesion suggestive of tuberculoma with positive Quantiferon - TB Gold test.

Keywords: exudative retinal detachment, orbital cellulitis, tuberculosis

\section{INTRODUCTION}

The term "ocular tuberculosis" is used to describe infections caused by Mycobacterium tuberculosis or any of the three other Mycobacteria species (sp. bovis, africanum, and microti) in the eye. The bacteria affect the eye either by a direct invasion after haematogenous dissemination accompanied by local inflammation, or via a hypersensitivity reaction to the bacteria with a focus elsewhere in the body ${ }^{1}$. Proptosis, intermittent diplopia and headaches or extra-ocular muscle motility restriction may be the presenting complaint secondary to intracranial inflammation. Less commonly, intraocular tuberculosis may present as a large tuberculoma. These tuberculomas may be located anywhere, may mimic a tumor measuring 4 to $14 \mathrm{~mm}$ in size and generally present as a yellowish subretinal mass with surrounding exudative retinal detachment $^{2}$.

\section{CASE REPORT}

A 40 year old male presented with chief complaints of swelling of upper and lower eyelids with protrusion of the left eye for 3 days associated with sudden diminution of vision in left eye for 1 day. There was no history of trauma, insect bite, sinusitis or dental caries. He is a diagnosed case of Type II Membranoproliferative Glomerulonephritis diagnosed 6 months back by a renal biopsy. He had been taking oral steroids since then.

On examination, his visual acuity was RE 6/6 and LE Hand movement. Extraocular movements were restricted in all positions of gaze. Left eye axial proptosis of $4 \mathrm{~mm}$ was present. It was irreducible, and no alteration could be made by change of posture or pressure on the neck veins. No bruit was heard on auscultation. (Figure 1). 360 chemosis, conjunctival congestion and circumciliary

Correspondence: Pooja Karki, Nepal Eye

Hospital, Kathmandu., Nepal. Email: karkipooja@hotmail.com. 


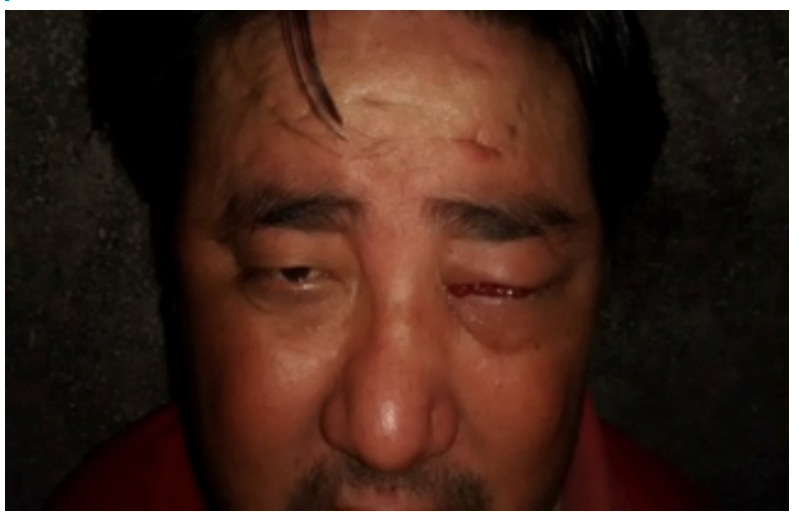

Figure 1.Clinical picture with gross proptosis

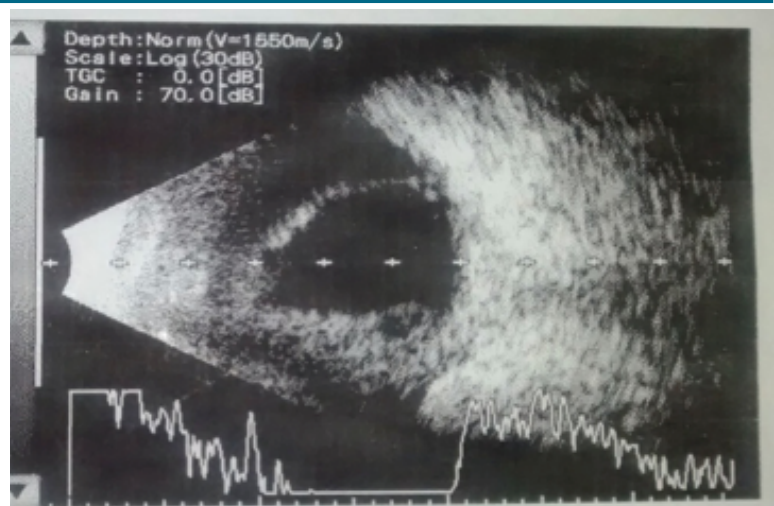

Figure 2. USG showing retinal detachment

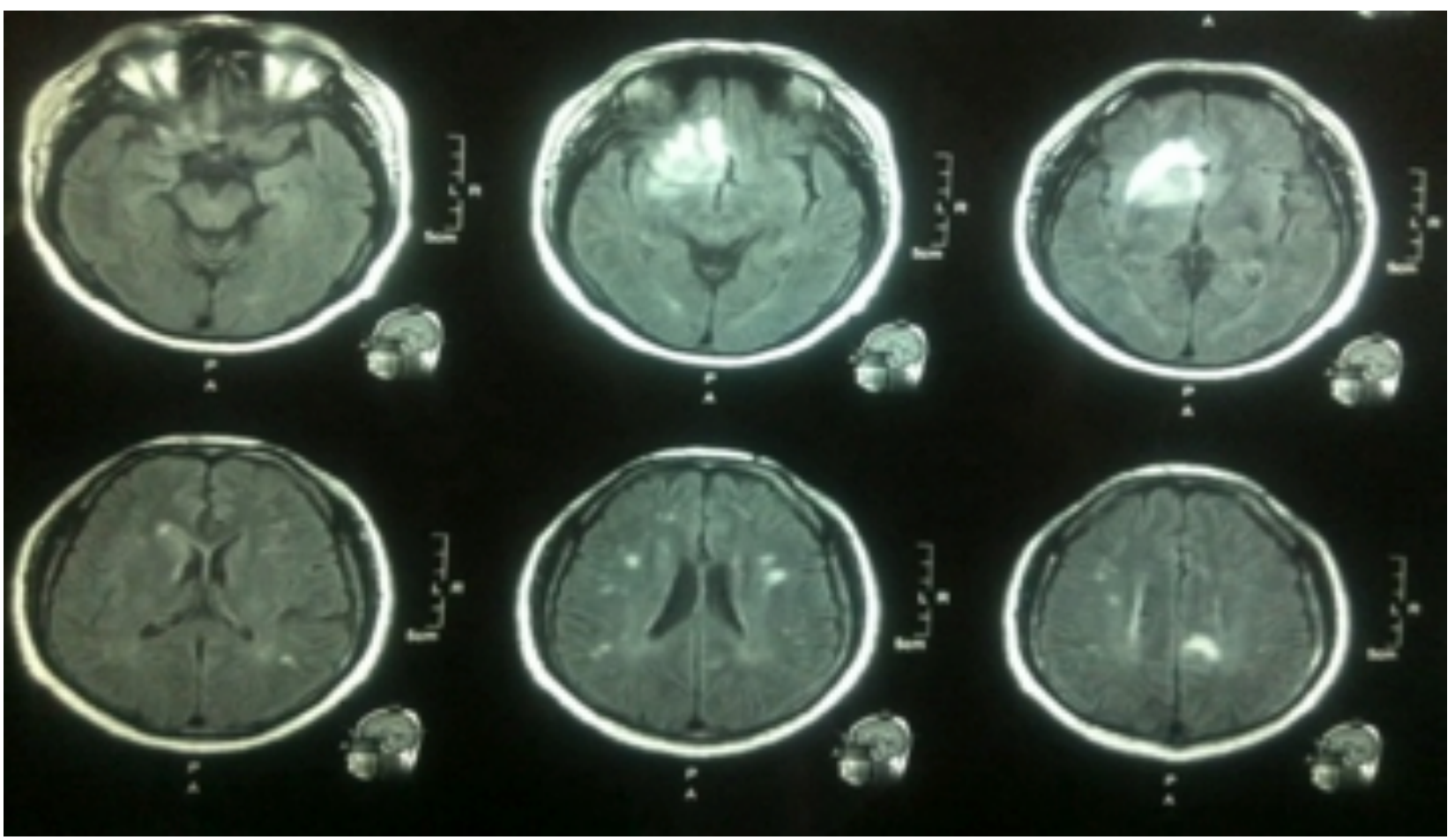

Figure 3: MRI of the brain showing cystic and infiltrating soft tissue mass in the right frontal lobe

congestion was present. Epithelial edema and stromal edema was present in the cornea. There were no iris nodules. Pupil was sluggishly reacting to both direct and consensual light. Grade 3+ vitreous cells were seen on posterior segment examination. Optic disc was normal. Exudative retinal detachment with subretinal abscess was present in the macular region as well as in inferior retinal and nasal retinal regions, all measuring more than 4 disc diameters. The total leucocyte count was 8,400 (neutrophils $75 \%$, lymphocytes-25\%). ESR was $25 \mathrm{~mm} / 1^{\mathrm{st}} \mathrm{hr}$. Blood urea was $68 \mathrm{mg} / \mathrm{dl}$ and serum creatinine was $2.4 \mathrm{mg} / \mathrm{dl}$. Chest X-ray was normal.

B-scan ultrasonography showed membrane like shadow in the vitreous with high reflectivity in A-scan suggestive of exudative retinal detachment (figure 2).

With a provisional diagnosis of Left orbital cellulitis grade II with subretinal abscess with exudative retinal detachment, patient was started on IV amoxicillin $1 \mathrm{gm}$ and clavulanate 
$200 \mathrm{mg}$ TDS. IV metronidazole $500 \mathrm{mg}$ TDS was also started. Topical antibiotics and cycloplegic were given. Tab acetazolamide $250 \mathrm{mg}$ TDS was given to lower the intraocular pressure. After the initiation of treatment proptosis gradually subsided but his vision deteriorated to perception of light (PL) with inaccurate projection of rays (PR).

MRI of the brain (figure 3) revealed cystic and infiltrating soft tissue mass in the right frontal lobe suggestive of tuberculoma. Left sided proptosis could also be seen.

Blood culture showed no growth of organisms. Neuro surgical consultation was done. Cerebrospinal fluid analysis showed protein $90 \mathrm{mg} \%$, glucose $60 \mathrm{mg} \%$. Quantiferon gold TB test was done which came out positive. Antitubercular therapy (ATT) was started on the evidence of clinical examination and investigations. Along with it oral steroids were also prescribed.

After 2 months of ATT his proptosis subsided. There were no subretinal abscess but the retina was still detached. His vision was still PL with inaccurate PR.

\section{DISCUSSION}

Thus far orbital cellulitis with exudative retinal detachment has been reported only a few times in literature 3,4,5. First case was reported in India in 1957 of a 12 year old boy. He had presented with orbital cellulitis with abscess and exudative retinal detachment. His condition had improved after the burst of the abscess and IV antibiotics. His visual acuity improved from $3 / 60$ to $6 / 9^{3}$. $2^{\text {nd }}$ case was reported in Pittsburg, USA in 2007 in a 59 year old patient with Down Syndrome. She had RE orbital cellulitis with RE exudative retinal detachment and choroidal detachment ${ }^{4}$. $3^{\text {rd }}$ case was reported in Athenes, Greece in 2014 in an 89 year old patient with Chronic Myeloid Leukemia. This patient had RE orbital cellulits with exudative retinal detachment. Patient improved after a course of IV antibiotics. Exudative retinal detachment resolved and vision improved from PL to 6/36 5 .

Type II MPGN also predisposes to exudative retinal detachment. In a study by mcavoy et al, among 26 biopsy proven cases of Type II MPGN one patient had exudative retinal detachment ${ }^{6}$.

Another study by Bajracharya et al, which comprised of 119 patients of chronic renal failure, there was one case of bilateral serous detachment ${ }^{7}$. Since our case had a subretinal absess, tuberculosis is more probable.

Orbital cellulitis is a relatively uncommon infectious process previously associated with severe complications involving ocular adnexal structures posterior to the orbital septum ${ }^{8}$. Sinusitis is the most common cause of orbital cellulitis followed by trauma. The most common microorganisms isolated from the drained abscesses are Staphylococci and Streptococci species 9

Tuberculosis of the orbit is rare. It is usually seen in the pediatric age-group. The disease is usually unilateral. The common presentations are proptosis, nontender or mildly painful orbital/lid swelling, and sinus formation. The predominant imaging features of orbital tuberculosis are involvement of the orbital bony wall and lacrimal gland, with soft tissue inflammatory mass/abscess formation ${ }^{10}$. Quantiferon-TB Gold In-Tube Test can 
differentiate the presence of latent TB from patients with positive skin tests due to vaccinations and those with negative skin tests due to allergy, such as occurs with sarcoidosis 11.

Ocular TB is treated as other forms of extrapulmonary TB. The treatment requires a bactericidal drug and a sterilizing agent. The first line antitubercular agents are isoniazid, rifampicin, pyrazinamide, streptomycin and ethambutol. Systemic steroids used for the first few weeks can be used with antitubercular treatment to decrease damage caused to ocular tissues. However, use of steroids alone should be avoided as it can flare up systemic tuberculosis and activate latent lesions ${ }^{12}$. Our patient also showed improvement after antitubercular therapy and oral steroids.

\section{REFERENCES}

1. Goyal JL, Jain P, Arora R, Dokania. Ocular manifestations of tuberculosis. Indian Journal of Tuberculosis. 2015; 62:66-73.

2. Sharma A, Thapa B, Lavaju P. Ocular tuberculosis: an update. Nepal J Ophthalmol. 2011; 3(5):52-67.

3. Malhotra M. Orbital cellulitis with exudative retinal detachment. Brit $\mathrm{J}$ Ophthal.1957; 41:317.

4. Farhi P, Kurup S Abdelghan W. Orbital cellulitis associated with combined retinal and choroidal detachments. Eye. 2007; 21:1009-10.

5. Farah E, Kalantzis G, Papaefthimiou I, Koutsandrea C, Georgalas I. Spontaneously resolved exudative retinal detachmentcaused by orbital cellulitis in an immunocompromised adult. Eye. 2014; 28:109-10.

6. McAvoy CE, Silvestri G. Retinal changes associated with type 2 glomerulonephritis. Eye. 2005; 19:985-9.

7. Bajracharya L, Shah DN, Raut KB, Koirala S. Ocular evaluation in patients with chronic renal failure -a hospital based study. Nepal Med Coll J 2008; 10(4): 209-14.

8. Connell B, Kamal Z, McNab AA. Fulminant orbital cellulitis with complete loss of vision. Clin Exp Ophthalmol. 2001; 29:260-1.

9. Chaudhary IA, Shamsi FA, Elzaridi E, AlRashed W, Al-Amri A, Al-Anezi F, Arat YO, Holck DE. Outcome of treated orbital cellulitis in a tertiary eye care center in the middle East. Ophthalmology. 2007; 114(2): 345-54.

10. Narula MK, Chaudhary V, Baruah D, Kathuria M, Anand R. Pictorial essay: Orbital tuberculosis. Indian $\mathrm{J}$ Radiol Imaging. $2010 \mathrm{Feb}$; 20(1):6-10.

11. Rangel CM, Atencia C, Merayo-Lloves J, Fernandez-Vega Sanz A. Presumed latent ocular tuberculosis diagnosed with the positive quantiFERON-TB Gold In-Tube Test in a HLA-A29-positive patient. BMJ Case Rep. 2015. Doi: 10.1136/bcr-2015210206 